\title{
Neonatal outcomes among twins stratified by method of conception: secondary analysis of maternal fetal medicine (MFMU) network database
}

\author{
Jaimin S. Shah ${ }^{1} \cdot$ Susan Hosseini Nasab ${ }^{1} \cdot$ Neil Chappell $^{2} \cdot$ Han-Yang Chen ${ }^{1} \cdot$ Amy Schutt $^{2} \cdot$ Hector Mendez-Figueroa $^{3}$
}

Received: 23 November 2017 / Accepted: 25 February 2018 / Published online: 9 March 2018

(C) Springer Science+Business Media, LLC, part of Springer Nature 2018

\begin{abstract}
Purpose To investigate whether twin pregnancies conceived by different forms of fertility treatments are associated with adverse neonatal outcomes and to examine the difference in maternal and obstetrical characteristics between patients.

Methods Our study was a retrospective analysis of twin pregnancies conceived by fertility treatments from a prospectively collected database. Treatments were stratified into two groups: group 1 (ART) consisted of in vitro fertilization (IVF) and intracytoplasmic sperm injection (ICSI), and group 2 (non-ART) included intrauterine insemination (IUI) and ovulation induction (OI). Composite neonatal morbidity included respiratory distress syndrome, intraventricular hemorrhage, leukomalacia, chronic lung disease, and death prior to discharge.

Results There were 460 neonates in our study; among them, 67\% $(n=310)$ were in group 1, and 33\% $(n=150)$ in group 2. Group 1 patients were more likely to be older $(p=0.004)$, nulliparous $(p=0.01)$, delivered twins with lower birth weights $(2278 \mathrm{~g} \pm 605$ vs. $2427 \pm 519, p=0.009)$, and had more deliveries $<32$ weeks gestation $(p=0.001)$. In multivariable Poisson regression model, only neonatal intensive care unit admission rate was increased for group 1 twins (aRR $=1.27,95 \%$ CI 1.003-1.60).

Conclusions After adjusting for confounders, twins conceived via ART compared to non-ART had similar neonatal outcomes. These data can help when counseling this patient population and assist in planning larger prospective cohorts.
\end{abstract}

Keywords Assisted reproductive technology $\cdot$ Twin gestation $\cdot$ Composite neonatal morbidity $\cdot$ Method of conception $\cdot$ Neonatal

\section{Introduction}

Increasing age of women at their first pregnancy is associated with higher rates of infertility and therefore, utilization of different fertility treatments may be warranted making twin pregnancies more common [1-3]. Several studies have reported higher adverse neonatal outcomes and differences in maternal

Jaimin S. Shah

Jaimin.Shah@uth.tmc.edu

1 Department of Obstetrics \& Gynecology, McGovern Medical School at the University of Texas Health Science Center, 6431 Fannin St, Suite 3.214, Houston, TX 77030, USA

2 Department of Reproductive Endocrinology and Infertility, Baylor College of Medicine, Houston, TX, USA

3 Department of Maternal \& Fetal Medicine, McGovern Medical School at the University of Texas Health Science Center, Houston, TX, USA characteristics in pregnancies conceived via assisted reproductive technology (ART) compared to spontaneous conception (SC) [4-8], while others have shown comparable results [9-11]. However, most reports have focused on singleton pregnancies. Few studies have investigated the maternal characteristics and neonatal outcomes specifically in twin gestations resulting from ART [10, 12-14].

When comparing dichorionic twin gestations conceived via ART compared to SC twins, studies have shown increased neonatal risks and differences in maternal characteristics for the ART group $[2,10,12,15,16]$. However, others have shown similar neonatal outcomes and maternal characteristics in twin gestations conceived via ART compared to SC [1, 9, 17-19]. Possible explanations for varying results could have been from misclassifying intrauterine insemination (IUI) and ovulation induction $(\mathrm{OI})$ into the $\mathrm{SC}$ twin gestation group $[8,20,21]$ or including IUI into the ART group [15]. Other studies have excluded OI or IUI from their studies and only include in vitro fertilization (IVF) or intracytoplasmic sperm injection (ICSI) 
[12]. These studies may misrepresent the infertile population, and the conclusions may be less generalizable. In addition, conflicting results may be from studies only adjusting for some confounding variables or none at all [9, 10, 22, 23].

There is a paucity of literature specifically addressing maternal characteristics and neonatal outcomes among twins conceived by different fertility treatments. Given that studies have shown differences in non-fertile vs. fertile patients (e.g., ART compared to SC twin pregnancies) [2, 10, 12, 15, 16], there might be differences, though less pronounced, when comparing twin pregnancies conceived via ART to less invasive fertility treatments (IUI/OI). Underlying infertility diagnosis can affect neonatal outcomes and maternal/delivery characteristics, but the type of fertility treatment utilized may not. This study focuses on patients requiring a fertility treatment and to see if there are differences by treatment type. The primary aim of this study was to investigate whether twin pregnancies conceived by different forms of fertility treatments (IVF/ICSI vs. IUI/OI) have an association with adverse neonatal outcomes. The secondary aim was to examine the differences in maternal and obstetrical characteristics between patients receiving different fertility treatments. We hypothesize that there will be no significant difference in maternal and obstetrical characteristics or neonatal outcomes when comparing between different fertility treatments.

\section{Materials and methods}

This study is a secondary analysis of a placebo-controlled, double-blinded, randomized clinical trial of 17 alphahydroxyprogesterone caproate (17P) for the prevention of preterm birth in multiple gestations [24]. This parent trial included 14 academic sites across the USA from 2004 to 2006. Briefly, gravid women with multiple gestation pregnancies between 16 and 20 weeks gestation were randomized to receive $17 \mathrm{P}$ or placebo. The only inclusion criterion was being a multiple gestation, so the baseline risk of preterm labor or birth was the same for all patients. The primary outcome was delivery or fetal death before 35 weeks of gestation. Treatment did not reduce the rate of preterm birth in women with twins.

In the initial study, data were collected on the method of conception of all patients. Patients either had spontaneous, IVF/ICSI, or IUI/OI conceived twins. No missing data was noted. For this analysis, all SC twin pregnancies, <20-week deliveries, or twin pregnancies with only one birth reported were excluded. No fetal malformations were noted. In our study, patients were included if they received a type of fertility treatment to conceive. We then stratified the patients into two groups. Group 1 included twin pregnancies conceived via ART (IVF/ICSI), and group 2 included twins conceived via non-ART (IUI/OI).
The primary neonatal outcome was the rate of composite neonatal morbidity (CNM), defined as any of the following outcomes: respiratory distress syndrome (RDS), intraventricular hemorrhage grades 3 and 4 (IVH), periventricular leukomalacia (PVL), bronchopulmonary dysplasia (BPD), and/or death prior to discharge. Secondary neonatal outcomes included neonatal intensive care unit (NICU) admission, RDS, mechanical ventilation (MV), sepsis, retinopathy of prematurity stage III or higher (ROP), necrotizing enterocolitis stages II and III (NEC), birth weight (BW), stillbirth, small for gestational age (GA), and neonatal death. All neonatal outcomes defined in our study followed the parent trial.

STrengthening the Reporting of OBservational studies in Epidemiology (STROBE) [25] guidelines were followed in our study. Descriptive statistics were used to report all variables of interest. Continuous variables were analyzed using student's $t$ test, while categorical variables used chi-square and Fisher's exact test. A $p$ value $<0.05$ was considered statistically significant. Multivariable Poisson regression models with robust error variance [26] were used to determine the association between ART technique and perinatal outcomes, while adjusting for maternal age, race/ethnicity, level of education, nulliparity, pre-pregnancy body mass index, and GA at time of delivery. The results were presented as adjusted relative risk (aRR) with accompanying 95\% confidence intervals (CIs). All analyses were run on STATA version 14 (College Station, TX). The study was considered exempt (HSC-MS17-0019) from the University of Texas Health Science Center McGovern Medical School Committee for the Protection of Human Subjects.

\section{Results}

In the parent trial, a total of 658 participants (1316 twins) were included in the study which is shown in Fig. 1. The final study population included 460 twins (230 pregnancies) conceived by a fertility treatment. Group 1 (67\%) included 310 twins (155 pregnancies) conceived via ART, while group $2(33 \%)$ included 150 twins (75 pregnancies) conceived via non-ART (Fig. 1). There was no significant difference $(p=0.14)$ in $17 \mathrm{P}$ use among both groups. Group 1 mothers were more likely to be older and nulliparous (Table 1).

Overall, the majority of twins in both groups were delivered by patients with a dichorionic placenta (Table 2). Group 1 twins were more likely to be delivered at an earlier GA compared to group 2 (Table 2). There was no significant difference in betamethasone use for fetal lung maturity or delivery route between the two groups (Table 2). Group 1 twins had a significantly higher rate of a NICU admission, while group 2 had a significantly higher rate of BPD and ROP (Table 3). In addition, group 1 twins had a significantly lower mean BW of almost $150 \mathrm{~g}$ when compared to group 2 (Table 3 ). 
Fig. 1 Inclusion criteria and flow chart of study population

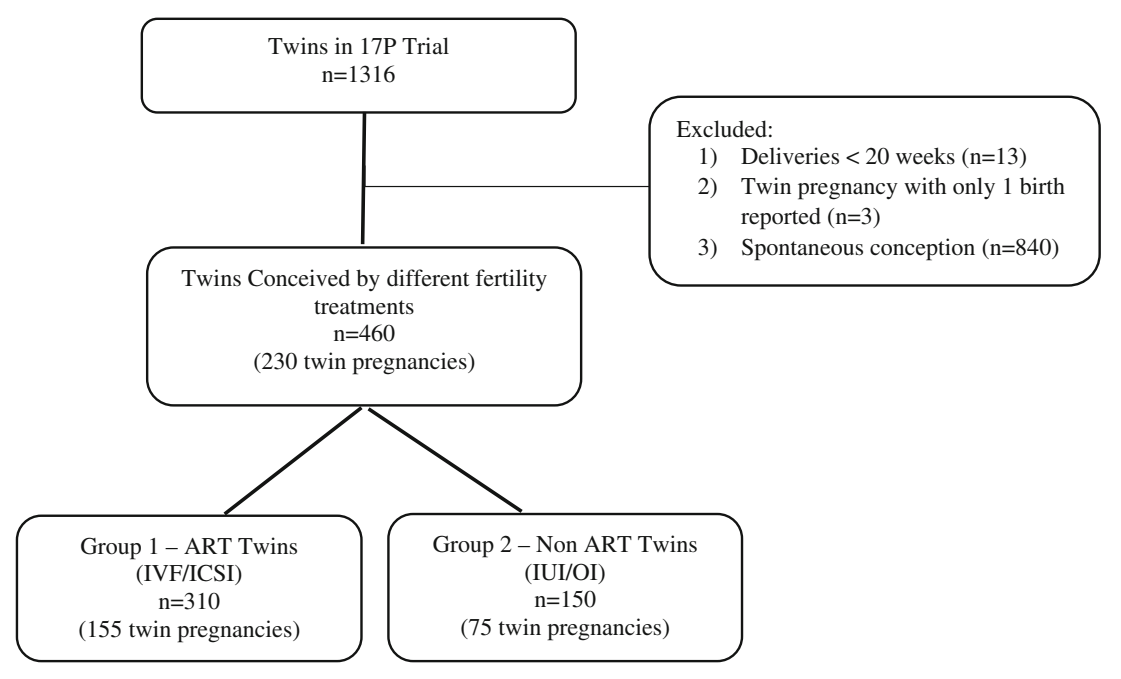

Table 1 Maternal characteristics by fertility treatments

\begin{tabular}{|c|c|c|c|}
\hline Maternal demographics & Group 1: IVF/ICSI $(n=155)$ & Group 2: IUI/OI $(n=75)$ & $p$ value \\
\hline Maternal age (years) & & & 0.004 \\
\hline $20-34$ & $68(43.9)$ & $48(64.0)$ & \\
\hline$\geq 35$ & $87(56.1)$ & $27(36.0)$ & \\
\hline Race/ethnicity & & & 0.40 \\
\hline Non-Hispanic White & $137(88.4)$ & $68(90.7)$ & \\
\hline Non-Hispanic Black & $5(3.2)$ & $3(4.0)$ & \\
\hline Hispanic & $4(2.6)$ & $3(4.0)$ & \\
\hline Non-Hispanic Other & $9(5.8)$ & $1(1.3)$ & \\
\hline Education & & & 0.99 \\
\hline$<12$ years & $1(0.6)$ & $0(0)$ & \\
\hline 12 years & $8(5.2)$ & $4(5.3)$ & \\
\hline$>12$ years & $146(94.2)$ & $71(94.7)$ & \\
\hline Married & $153(98.7)$ & $75(100)$ & 0.99 \\
\hline Nulliparous & $107(69.0)$ & $39(52.0)$ & 0.01 \\
\hline Pre-pregnancy BMI & & & 0.39 \\
\hline$<25$ & $97(62.6)$ & $48(64.0)$ & \\
\hline $25 \leq 30$ & $35(22.6)$ & $12(16.0)$ & \\
\hline$\geq 30$ & $23(14.8)$ & $15(20.0)$ & \\
\hline Smoking during pregnancy & $2(1.3)$ & $0(0)$ & 0.99 \\
\hline Preterm labor & $66(42.6)$ & $31(41.3)$ & 0.86 \\
\hline Antepartum bleeding & $2(1.3)$ & $1(1.3)$ & 0.99 \\
\hline Number of miscarriage & & & 0.70 \\
\hline 0 & $110(71.0)$ & $50(66.7)$ & \\
\hline $1-2$ & $41(26.5)$ & $22(29.3)$ & \\
\hline$\geq 3$ & $4(2.6)$ & $3(4.0)$ & \\
\hline Chorioamnionitis & $6(3.9)$ & $0(0)$ & 0.18 \\
\hline $\mathrm{BV} / \mathrm{CT} /$ trichomonas & $1(0.6)$ & $0(0)$ & 0.99 \\
\hline Group B strep and/or herpes & $2(1.3)$ & $0(0)$ & 0.99 \\
\hline UTI/pyelonephritis & $12(7.7)$ & $6(8.0)$ & 0.95 \\
\hline
\end{tabular}

Data are presented as $n(\%)$

$B V$, bacterial vaginosis; $C T$, chlamydia; $B M I$, body mass index $\left(\mathrm{kg} / \mathrm{m}^{2}\right) ; U T I$, urinary tract infection 
Table 2 Obstetrical and delivery characteristics by fertility treatments

\begin{tabular}{|c|c|c|c|}
\hline Obstetrical and delivery characteristics & $\begin{array}{l}\text { Group 1: IVF/ICSI } \\
(n=310)\end{array}$ & $\begin{array}{l}\text { Group 2: IUI/OI } \\
(n=150)\end{array}$ & $p$ value \\
\hline Gestational age at time of delivery (weeks) & & & 0.001 \\
\hline$<28$ & $10(3.2)$ & $5(3.3)$ & \\
\hline $28-31$ & $30(9.7)$ & $2(1.3)$ & \\
\hline$\geq 32$ & $270(87.1)$ & $143(95.3)$ & \\
\hline Delivery route & & & 0.46 \\
\hline Cesarean delivery & $189(61.4)$ & $86(57.7)$ & \\
\hline Vaginal delivery & 119 (38.6) & $63(42.3)$ & \\
\hline Reason for cesarean delivery & & & 0.006 \\
\hline $\mathrm{CPD}$, failed induction, abnormal presentation & $104(55.6)$ & $49(57.0)$ & \\
\hline Non-reassuring fetal status, cord prolapse & $9(4.8)$ & $6(7.0)$ & \\
\hline Previous cesarean delivery & $8(4.3)$ & $12(14.0)$ & \\
\hline Elective & $4(2.1)$ & $2(2.3)$ & \\
\hline Other & $32(17.1)$ & $6(7.0)$ & \\
\hline Treated with betamethasone & $70(22.6)$ & $38(25.3)$ & 0.51 \\
\hline Type of labor & & & 0.03 \\
\hline Spontaneous & $119(39.0)$ & $58(38.9)$ & \\
\hline Induced & $63(20.7)$ & $46(30.9)$ & \\
\hline No labor & $123(40.3)$ & $45(30.2)$ & \\
\hline Length of labor (spontaneous vaginal deliveries) & & & 0.24 \\
\hline $0 \leq 8 \mathrm{~h}$ & $45(62.5)$ & $14(48.3)$ & \\
\hline $8-24 \mathrm{~h}$ & $25(34.7)$ & $15(51.7)$ & \\
\hline$>24 \mathrm{~h}$ & $2(2.8)$ & $0(0)$ & \\
\hline Reason for induction (if induced) & & & 0.12 \\
\hline Maternal indications & $26(41.9)$ & $24(52.2)$ & \\
\hline Fetal indications & $12(19.4)$ & $14(30.4)$ & \\
\hline Premature rupture of membranes & $6(9.7)$ & $2(4.3)$ & \\
\hline Elective & $18(29.0)$ & $6(13.0)$ & \\
\hline Length of rupture & & & 0.19 \\
\hline $0 \leq 8 \mathrm{~h}$ & $108(62.1)$ & $70(72.9)$ & \\
\hline $8-24 \mathrm{~h}$ & $54(31.0)$ & $22(22.9)$ & \\
\hline$>24 \mathrm{~h}$ & $12(6.9)$ & $4(4.2)$ & \\
\hline Dichorionic twins & $298(96.1)$ & $146(97.3)$ & 0.60 \\
\hline
\end{tabular}

Data are presented as $n(\%)$

$C P D$, cephalopelvic disproportion
After adjusting for confounders, group 1 twins had a significant increased aRR for a NICU admission (Table 4). However, there was no significant difference in aRR for ROP and BPD, and all remaining neonatal outcomes were similar between groups (Table 4).

\section{Conclusions}

In this retrospective secondary analysis of a randomized clinical trial data, we observed that the majority of the maternal characteristics, obstetrical and delivery characteristics, and neonatal outcomes were similar between twins conceived by IVF/ICSI compared to IUI/OI. The lower BW for group 1 twins likely is associated to the increased risk of delivering at an earlier GA. In addition, the increase in NICU admission rate for group 1 twins could not be accounted for by the adjusted confounders. The increased risk of a NICU admission in group 1 may be in part to an earlier GA at delivery and lower BW. Also, there might be a bias towards delivery at an earlier GA and more frequent NICU admissions because of an ART conception. Patients in group 1 may have had a worse prognosis or already failed less invasive treatments. 
Table 3 Neonatal outcomes by fertility treatments

\begin{tabular}{|c|c|c|c|}
\hline Neonatal outcomes & Group 1: IVF/ICSI $(n=310)$ & Group 2: IUI/OI $(n=150)$ & $p$ value \\
\hline Female infant & $162(53.5)$ & $77(51.7)$ & 0.72 \\
\hline Birth weight & & & 0.009 \\
\hline$<2000 \mathrm{~g}$ & $89(29.4)$ & $28(18.8)$ & \\
\hline $2000-2499 \mathrm{~g}$ & $104(34.3)$ & $46(30.9)$ & \\
\hline$\geq 2500 \mathrm{~g}$ & $110(36.3)$ & $75(50.3)$ & \\
\hline Birth weight (g) mean (SD) & $2278( \pm 605)$ & $2427( \pm 519)$ & 0.01 \\
\hline Composite neonatal morbidity & $36(11.9)$ & $9(6.0)$ & 0.051 \\
\hline Small for gestational age & $26(8.6)$ & $7(4.7)$ & 0.14 \\
\hline Apgar $\leq 3$ at $1 \mathrm{~min}$ & $21(7.0)$ & $5(3.4)$ & 0.12 \\
\hline Apgar $\leq 3$ at $5 \mathrm{~min}$ & $6(2.0)$ & $1(0.7)$ & 0.43 \\
\hline NICU Admission & $154(50.8)$ & $57(38.3)$ & 0.01 \\
\hline Retinopathy of prematurity & $0(0)$ & $3(2.0)$ & 0.04 \\
\hline Intraventricular hemorrhage III/IV & $1(0.3)$ & $0(0)$ & 0.99 \\
\hline Periventricular leukomalacia & $1(0.3)$ & $0(0)$ & 0.99 \\
\hline Necrotizing enterocolitis stage II/III & $1(0.3)$ & $0(0)$ & 0.99 \\
\hline Respiratory distress syndrome & $28(9.2)$ & $9(6.0)$ & 0.24 \\
\hline Bronchopulmonary dysplasia & $2(0.7)$ & $6(4.0)$ & 0.02 \\
\hline Sepsis & $4(1.3)$ & $3(2.0)$ & 0.69 \\
\hline Mechanical ventilation & $22(7.3)$ & $11(7.4)$ & 0.96 \\
\hline Transient tachypnea of the newborn & $49(16.3)$ & $19(12.8)$ & 0.33 \\
\hline Vanishing fetus & $24(7.7)$ & $6(4.0)$ & 0.13 \\
\hline Definite seizure & $1(0.3)$ & $1(0.7)$ & 0.55 \\
\hline Death before discharge & $5(1.7)$ & $1(0.7)$ & 0.67 \\
\hline Stillbirth & $5(1.6)$ & $0(0)$ & 0.18 \\
\hline Meconium aspiration syndrome & $1(0.3)$ & $0(0)$ & 0.99 \\
\hline
\end{tabular}

Data are presented as $n(\%)$

NICU, neonatal intensive care unit; $C N M$, respiratory distress syndrome, intraventricular hemorrhage grades 3 and 4, periventricular leukomalacia, bronchopulmonary dysplasia, and/or death prior to discharge
In a systematic review and meta-analysis, Qin et al. reported higher adverse outcomes such as placenta previa, elective cesarean section, preterm birth, very preterm birth, lower mean BW, and congenital malformations in dichorionic twin gestations conceived via ART compared to SC twins [12]. However, some studies included in their review did not adjust for confounding variables, classified OI and IUI into the SC twin category, or did not include OI or IUI at all in their
Table 4 Adjusted relative risk between fertility treatments and neonatal outcomes

\begin{tabular}{ll}
\hline Neonatal outcomes & Adjusted relative risk* $(95 \% \mathrm{CI})$ \\
\hline Small for gestational age & $1.45(0.65-3.25)$ \\
Apgar $\leq 3$ at $1 \mathrm{~min}$ & $1.62(0.59-4.41)$ \\
Apgar $\leq 3$ at $5 \mathrm{~min}$ & $2.03(0.17-24.62)$ \\
NICU admission & $1.27(1.003-1.60)$ \\
Respiratory distress syndrome & $1.07(0.53-2.14)$ \\
Transient tachypnea of the newborn & $1.32(0.80-2.19)$ \\
Vanishing fetus & $1.45(0.58-3.64)$ \\
Composite neonatal morbidity & $1.34(0.72-2.49)$ \\
\hline
\end{tabular}

NICU, neonatal intensive care unit

*Adjusted for age, race/ethnicity, level of education, nulliparity, body mass index, gestational age at time of delivery 
analysis [12]. Moini et al. compared only IVF or ICSI to SC twin pregnancies [10]; they reported similar obstetrical but increased adverse neonatal outcomes for ART pregnancies such as NICU admission rate and perinatal mortality. Morcel et al. compared spontaneously and non-spontaneously (IVF, ICSI, IUI, and OI) conceived twins [15]; after adjusting for maternal age and parity, the non-spontaneous group showed an increased risk of very preterm birth, low and very low BW, NICU admission, and fetal or neonatal death rate. Also, when removing OI and adjusting for age and parity, ART (IVF, ICSI, IUI) compared to spontaneously conceived twins showed no significant differences in the maternal or perinatal outcomes [15]. However, OI conceived twins compared to SC twins showed a significant increase in preterm and very preterm births, very low BW, NICU admission, and fetal or neonatal death rates [15].

Studies by Domingues et al., Bensdorp et al., and Geisler et al. showed no increase in neonatal outcomes when comparing ART vs. SC twins [1, 16, 17]. In contrast to our study, Domingues et al. only adjusted for chorionicity and age [1]; Bensdorp et al. adjusted for maternal age, ethnicity, socioeconomic status, and fetal gender [17]; while Geisler et al. adjusted for maternal age, parity, and type of antenatal care received (public or private) [16]. Similar to our study, Bensdorp et al. included all four types of fertility treatment, while Domingues et al. and Geisler et al. did not include IUI and IUI/OI in their analysis, respectively $[1,16$, 17]. These studies also used SC twins as a reference group, while our study specifically compared the different types of fertility treatments between themselves.

Our study has several strengths. There has been limited research evaluating maternal characteristics and neonatal outcomes when comparing the different forms of fertility treatments for twin conception. We included all four categories of fertility treatments in our study, while majority of prior studies did not include all treatment options $[1,8,12]$. Our study was a secondary analysis of a multicenter prospectively collected data set by trained personnel. This allowed for a heterogeneous population and a more likely representation of patients receiving fertility treatments nationwide; many studies were solely at one institution. This study had a relatively large sample size, reviewed a variety of neonatal outcomes, and was more in tune with modern obstetrical practices.

Several limitations, however, need to be acknowledged. Due to a limited sample size, subgroup analyses of neonatal outcomes stratified by GA were not feasible. However, our analysis of neonatal outcomes adjusted for several potential confounders, including GA at time of delivery, maternal age, race/ethnicity, level of education, nulliparity, and prepregnancy body mass index. Group 1 had twice as many twins compared to group 2 and had older nulliparous patients. Group 1 also had higher "no labor" and cesarean delivery rates with a higher incidence of GA less than 32 weeks, which may be related to, as well as impact, outcome. Our original data set did not include infertility etiology, fertility treatment interventions, duration of infertility treatment, complications during treatments, or fertility treatment medications used. In addition, it was not specified if patients used fresh or frozen embryos or donor oocytes/sperm. It is known that BW is lower in fresh vs. frozen embryo transfers in both singletons and twins [27]. Medications for OI or reason for undergoing fertility treatment were not collected. A baseline difference in infertility etiology may be the reason why some patients received IVF/ICSI or IUI/ OI. While the majority of IUIs are typically in concert with OI, it cannot be stated with certainty if patients in group 2 received both IUI and OI treatments. It is a weakness inherent to a retrospective cohort, but the study comprises a large number of twin pregnancies to try to accommodate the possible heterogeneous nature of the cohort. Given this subgroup analysis was of patients that were randomized for another indication, the subgroups may not be equivalent at baseline. Future studies are warranted to investigate differences in each fertility treatment with a larger sample size and consider comparing differences by infertility diagnosis and treatment received.

Antenatal care and obstetrical management likely varied from the different institutions, which might play a role in influencing outcomes. Differences in infertility laboratories and IVF/ICSI/IUI protocols might have played a contributing factor. Therefore, there is potential of unmeasured confounding. In addition, the rare occurrence of some adverse neonatal outcomes also may not allow for certain associations with fertility treatment to become evident. Since the majority of patients had dichorionic placentas, only a small portion of monochorionic placentas were included in our study which may have affected the results given the increased risks for monochorionic twin gestations. Future research is needed to assess the effects of chorionicity between the different fertility treatment options.

In conclusion, our study demonstrated twins conceived via ART compared to non-ART had mostly similar maternal and obstetrical characteristics and neonatal outcomes. Providers managing patients who require fertility treatments to conceive can counsel and make them aware of a slightly increased risk of lower BW, earlier GA at delivery, and a higher rate of NICU admissions in ART compared to non-ART. However, providers can impart reassurance to their patients given overall outcomes were similar in patients receiving a fertility treatment. This information may be applicable to not only infertility specialists but also obstetricians and NICU providers. Our study can assist in planning larger cohorts to confirm our findings when specifically comparing twin pregnancies conceived by a fertility treatment.

\section{Compliance with ethical standards}

Conflicts of interest The authors declare that they have no conflict of interest. 


\section{References}

1. Domingues AP, Dinis SR, Belo A, Couto D, Fonseca E, Moura P. Impact of induced pregnancies in the obstetrical outcome of twin pregnancies. Fertil Steril. 2014;101:172-7.

2. Bamberg C, Fotopoulou C, Neissner P, Slowinski T, Dudenhausen JW, Proquitte $\mathrm{H}$, et al. Maternal characteristics and twin gestation outcomes over 10 years: impact of conception methods. Fertil Steril. 2012;98:95-101.

3. Coetsier T, Devroey P, Dhont M, Edwards R, Evers H, Hagglund L, et al. Prevention of twin pregnancies after IVF/ICSI by single embryo transfer. Hum Reprod. 2001;16:790-800.

4. Henningsen AKA, Pinborg A. Birth and perinatal outcomes and complications for babies conceived following ART. Semin Fetal Neonatal Med Elsevier Ltd. 2014;19:234-8.

5. Talaulikar VS, Arulkumaran S. Maternal, perinatal and long-term outcomes after assisted reproductive techniques (ART): implications for clinical practice. Eur J Obstet Gynecol Reprod Biol. 2013;170:13-9.

6. Zhu L, Zhang Y, Liu Y, Zhang R, Wu Y, Huang Y, et al. Maternal and live-birth outcomes of pregnancies following assisted reproductive technology: a retrospective cohort study. Sci Rep. 2016;6: 35141.

7. Jeve YB, Potdar N, Opoku A, Khare M. Donor oocyte conception and pregnancy complications: a systematic review and meta-analysis. BJOG An Int J Obstet Gynaecol. 2016;123:1471-80.

8. Pinborg A, Loft A, Rasmussen S, Schmidt L, Langhoff-Roos J, Greisen G, et al. Neonatal outcome in a Danish national cohort of 3438 IVF/ICSI and 10362 non-IVF/ICSI twins born between 1995 and 2000. Hum Reprod. 2004;19:435-41.

9. Vasario E, Borgarello V, Bossotti C, Libanori E, Biolcati M, Arduino S, et al. IVF twins have similar obstetric and neonatal outcome as spontaneously conceived twins: a prospective followup study. Reprod BioMed Online. 2010;21:422-8.

10. Moini A, Shiva M, Arabipoor A, Hosseini R, Chehrazi M, Sadeghi $\mathrm{M}$. Obstetric and neonatal outcomes of twin pregnancies conceived by assisted reproductive technology compared with twin pregnancies conceived spontaneously: a prospective follow-up study. Eur J Obstet Gynecol Reprod Biol. 2012;165:29-32.

11. Poon WB, Lian WB. Perinatal outcomes of intrauterine insemination/clomiphene pregnancies represent an intermediate risk group compared with in vitro fertilisation/intracytoplasmic sperm injection and naturally conceived pregnancies. J Paediatr Child Health. 2013;49:733-40.

12. Qin JB, Wang H, Sheng X, Xie Q, Gao S. Assisted reproductive technology and risk of adverse obstetric outcomes in dichorionic twin pregnancies: a systematic review and meta-analysis. Fertil Steril. 2016;105:1180-92.

13. Pinborg A. IVF/ICSI twin pregnancies: risks and prevention. Hum Reprod Update. 2005;11:575-93.

14. Caserta D, Bordi G, Stegagno M, Filippini F, Podagrosi M, Roselli $\mathrm{D}$, et al. Maternal and perinatal outcomes in spontaneous versus assisted conception twin pregnancies. Eur J Obstet Gynecol Reprod Biol. 2014;174:64-9.

15. Morcel K, Lavoué V, Beuchée A, Le Lannou D, Poulain P, Pladys P. Perinatal morbidity and mortality in twin pregnancies with dichorionic placentas following assisted reproductive techniques or ovarian induction alone: a comparative study. Eur J Obstet Gynecol Reprod Biol. 2010;153:138-42.

16. Geisler ME, O’Mahony A, Meaney S, Waterstone JJ, O’Donoghue $\mathrm{K}$. Obstetric and perinatal outcomes of twin pregnancies conceived following IVF/ICSI treatment compared with spontaneously conceived twin pregnancies. Eur J Obstet Gynecol Reprod Biol. 2014;181:78-83.

17. Bensdorp AJ, Hukkelhoven CW, van der Veen F, Mol BWJ, Lambalk CB, van Wely M. Dizygotic twin pregnancies after medically assisted reproduction and after natural conception: maternal and perinatal outcomes. Fertil Steril. 2016:371-377.e2.

18. Boulet SL, Schieve LA, Nannini A, Ferre C, Devine O, Cohen B, et al. Perinatal outcomes of twin births conceived using assisted reproduction technology: a population-based study. Hum Reprod. 2008;23:1941-8.

19. Sciscione A. Perinatal outcomes in women with twin gestations who conceived spontaneously versus by assisted reproductive techniques (ART). Am J Obstet Gynecol. 2007;197:S84.

20. Smithers PR, Halliday J, Hale L, MacKenzie Talbot J, Breheny S, Healy D. High frequency of cesarean section, antepartum hemorrhage, placenta previa, and preterm delivery in in-vitro fertilization twin pregnancies. Fertil Steril. 2003;80:666-8.

21. Hansen M, Kurinczuk JJ, de Klerk N, Burton P, Bower C. Assisted reproductive technology and major birth defects in Western Australia. Obstet Gynecol. 2012;120:852-63.

22. Egic AS, Mojovic DV, Milovanovic ZM, Jurisic AB, Srbinovic LP, Krsmanovic SP, et al. Degree and rate of growth discordance in dichorionic twins conceived by in vitro fertilization. Obstet Gynecol Int. 2014;2014:543728.

23. Yang H, Choi YS, Nam KH, Kwon JY, Park YW, Kim YH. Obstetric and perinatal outcomes of dichorionic twin pregnancies according to methods of conception: spontaneous versus in-vitro fertilization. Twin Res Hum Genet. 2011;14:98-103.

24. Rouse DJ, Caritis SN, Peaceman AM, Sciscione A, Thom EA, Spong CY, et al. A trial of 17 alpha-hydroxyprogesterone caproate to prevent prematurity in twins. N Engl J Med. 2007;357:454-61.

25. von Elm E, Altman DG, Egger M, Pocock SJ, Gøtzsche PC, Vandenbroucke JP. The strengthening the reporting of observational studies in epidemiology (STROBE) statement: guidelines for reporting observational studies. Int J Surg. 2014;12:1495-9.

26. Zou G. A modified Poisson regression approach to prospective studies with binary data. Am J Epidemiol. 2004;159:702-6.

27. Ozgur K, Berkkanoglu M, Bulut H, Humaidan P, Coetzee K. Perinatal outcomes after fresh versus vitrified-warmed blastocyst transfer: retrospective analysis. Fertil Steril Elsevier Inc. 2015;104:899-907e3. 